\title{
On The Value of Alert Systems and Gentle Rule Enforcement in Addressing
}

\section{Pandemics}

\author{
Roth, Yefim ${ }^{1}$, Plonsky, Ori ${ }^{2}$, Shalev, Edith $^{3}$, and Erev, Ido $^{2}$
}

\begin{abstract}
${ }^{1}$ University of Haifa; ${ }^{2}$ Technion - Israel Institute of Technology; ${ }^{3}$ Interdisciplinary Center Herzliya

The recent COVID-19 pandemic poses a challenge to policy makers on how to make the population adhere to the social distancing and personal protection rules. The current research compares two ways by which tracking smartphone applications can be used to reduce the frequency of reckless behaviors that spread pandemics. The first involves the addition of alerts that increase the users' benefit from responsible behavior. The second involves the addition of a rule enforcement mechanism that reduces the users' benefit from reckless behavior. The effectiveness of the two additions is examined in an experimental study that focuses on an environment in which both additions are expected to be effective under the assumptions that the agents are expected-value maximizers, risk averse, behave in accordance with cumulative prospect theory (Tversky \& Kahneman, 1992), or behave in accordance with the Cognitive Hierarchy model (Camerer, Ho \& Chong, 2004). The results reveal a substantial advantage to the enforcement application. Indeed, the alerts addition was completely ineffective. The findings align with the small samples hypothesis, suggesting that decision makers tend to select the options that led to the best payoff in a small sample of similar past experiences. In the current context the tendency to rely on a small sample appears to be more consequential than other deviations from rational choice.
\end{abstract}

Keywords: Decisions from experience, rare-events, social networks, levels or reasoning, trust game. 
The success in fighting the spread of COVID-19 pandemic in China is attributed, at least in part, to aggressive use of tracking smartphone applications (apps). These apps allow authorities to identify and isolate those who might mitigate spreading of the virus (Huang, Sun \& Sui, 2020), and punish those who violate social distancing and personal protection rules. For example, the apps issue color codes - green, yellow, or red - that indicate whether the holder poses an infection-transmission risk. A green light that grants people unrestricted pass (e.g. to the subway, work office, and other public places) is essential for daily life. Yellow and especially red codes are extremely confining; both indicate that their holder should be quarantined and cannot travel from one place to another. Identifying a person traveling with a red code is a sufficient reason to call the police. Thus, to enforce public health regulations, authorities may severely penalize persons with yellow or red codes that break quarantine.

When COVID-19 had spread to western countries, their policy makers tried to emulate the success of China's tracking app. Yet, possibly due to privacy and civil rights concerns, authorities in many western democracies held back on developing aggressive and intrusive enforcement features. Instead, most tracking apps were designed to serve for alert purposes only, under the assumption that a reliable alert would suffice to discourage reckless behaviors. However, it is unclear whether virus-tracking apps, which only alert users and forgo regulation enforcement, are sufficiently effective in discouraging reckless behaviors.

The current research compares the effectiveness of two strategies that could guide the design of less aggressive, but potentially effective, tracking apps. The starting point of the current analysis is that the individual's risk to be infected in a pandemic depends not only on one's own risk-taking behavior, but also on others'. Managing life at the time of a pandemic presents risk even to those who maintain social distancing and other health protection guidelines. In that respect, health related behavior during pandemics is similar to driving; sharing the road with other drivers presents risk even to cautious drivers. To combat the virus, it is therefore essential to understand not only the individuals' risk-taking behaviors but also the social dynamics that may arise in such situations. For example, it is possible that a minority of agents who engage in reckless behaviors (behaviors that could increase the risk of infection) would turn other agents' effort to behave responsibly futile, and in so doing drive otherwise responsible agents to behave recklessly as well (Erev, Plonsky, \& Roth, 2020). ${ }^{1}$

This research focuses on an abstraction of a world in which a disaster event (large rare loss) like a coronavirus infection, can result from the reckless behavior of either the individual or the other agents. Specifically, we analyze a repeated play of variants of the 4-person 'Reckless or Responsible' game described in the upper panel of Table 1. This game models an environment in which reckless behavior is beneficial most of the time, but if none of the agents is reckless, behaving responsibly is the best choice on average.

37

38

39

\footnotetext{
${ }^{1}$ For instance, consider a cautious driver who prefers to slow down when a traffic light changes from green to orange. If the car behind does not show signs of slowing down, it is safer for the cautious driver to speed up and drive through the orange light to avoid an accident.
} 
1 Table 1: Three variations of the Reckless or Responsible game, predictions, and the observed 2 Reckless-rate

\begin{tabular}{cccc}
\multicolumn{3}{c}{ Predicted Reckless-rate } & \\
Cognitive & Naïve & SAW & \\
Hierarchy & sampler & $\kappa=9$ & Observed \\
$\tau=1.54$ & $\mathrm{k}_{\mathrm{i}}=5$ & $\omega=0.5$ & Reckless \\
& & $\varepsilon=0.4$ & rate \\
& &
\end{tabular}

(1) Basic setting:

Reckless: 1, 0.98; -60 otherwise $(\mathrm{EV}=-0.22)$

Responsible: 0 if all agents choose Responsible;

$0,0.98 ;-60$ otherwise (if at least one agent chooses

$\begin{array}{llll}0.89 & 0.91 & 0.82 & 0.91\end{array}$

Reckless)

(2) Protecting Alert app:

Reckless: $1,0.98$; -60 otherwise $(\mathrm{EV}=-0.22)$

Responsible: 0 with certainty

$\begin{array}{llll}0.11 & 0.91 & 0.78 & 0.92\end{array}$

(3) Enforcing app:

Reckless: $-0.2,0.98 ;-61.2$ otherwise $(\mathrm{EV}=-1.42)$

Responsible: 0 if all agents choose Responsible;

$0,0.98 ;-60$ otherwise (if at least one agent chooses

$\begin{array}{llll}0.11 & 0 & 0.13 & 0.15\end{array}$

Reckless)

Note. SAW = Sampling and Weighting model (see Appendix 1).

The basic game has two Nash equilibria (choice profiles in which no agent wants to change choice unilaterally): An efficient equilibrium in which all agents choose 'Responsible' and earn 0 with certainty, and an inefficient equilibrium whereby all agents choose 'Reckless' and suffer an expected loss of 0.22 . While agents should prefer the efficient equilibrium, at least two factors could impair coordination and drive them to the inefficient reckless equilibrium. The first is fear from (or the expectation of) reckless behavior of other agents. Agents that worry that the other agents will choose Reckless are expected to choose Reckless as well. Fear of this type is predicted, for example, by the popular "levels of reasoning" models of behavior in games (Stahl \& Wilson, 1995; Nagel, 1995; Costa-Gomez, Crawford \& Broseta 2001; Camerer, Ho \& Chong, 2004). Under such models, agents have some level of reasoning and play best-reply to lower levels. Specifically, some agents (who are "level-0") choose randomly and other agents (e.g. "level-1") choose the best reply to those playing level-0. Here, this best-reply implies being Reckless (as a result, higher level agents will also choose Reckless).

A second relevant factor is that decision makers tend to select the options that led to the best payoff in a small sample of similar past experiences (Plonsky et al., 2015; Roth et al., 
2016). ${ }^{2}$ In the basic game, this tendency implies high Reckless rate because small samples are not likely to include the rare loss. For example, the probability that a $2 \%$ event will be included in a random sample of size 5 is only 0.095 .

If agents act in line with the 'level of reasoning' or 'small samples' hypotheses, then most of the agents in the basic 'Reckless or Responsible' game will choose to act recklessly. In search for a strategy to encourage responsible behavior we examine two variations of the basic game. The first involves the elimination of the problematic social externalities that lead agents to expect a higher utility from reckless behavior. This solution implies the design of an app that protects the agent from the reckless behavior of other agents. For example, by sending alerts when approaching people who tend to exhibit reckless behavior. The second panel in Table 1 presents a variant of the basic game with the perfectly protecting Alert app. Under this solution, the reckless behavior of others does not affect those who choose Responsible because they adhered to the alert and avoided the risk of infection. Therefore, choosing Responsible maximizes expected value. Under the levels of reasoning hypothesis, the Alert app ensures that level-1 and more sophisticated agents will behave responsibly. In addition, Responsible choice minimizes risk and should be selected if losses loom larger than gains (Kahneman \& Tversky, 1979) and if the agents are risk averse.

The second solution involves gentle rule enforcement (Erev et al., 2010; Schurr, Rodensky \& Erev 2014) that gently penalizes reckless behaviors with high probability (without eliminating the social externalities). One way to implement gentle enforcement in a pandemic is to use tracking applications that continuously monitor a person's behavior, and recommend the avoidance (early termination) of detected reckless activities. For example, if the agent enters a crowded party, the app will ask him to go out. If the agent accepts the recommendation, the punishment for initially behaving recklessly is gentle, just missing the party (and wasting time going to the party's location). The lower panel in Table 1 presents a variant of the basic game that demonstrates this solution with a gentle punishment (loss of 1.2 points) for each Reckless choice. Under such regime, reckless behavior is never the best choice, and agents are expected to choose Responsible even if they rely on small samples.

The central columns in Table 1 present the predicted reckless rate in the current games under the most popular levels of reasoning model (Cognitive Hierarchy; Camerer et al., 2004) and two abstractions of the reliance on small samples hypothesis (see Appendix 1). The predictions of the Cognitive Hierarchy model were derived with the parameter proposed by Camerer et al. (2004). According to the basic naïve sampler model, agents would choose Responsible in the first trial, and then select the option that led to the best outcome in a random sample of five previous experiences (Erev \& Roth, 2014). The model SAW (Sampling and Weighting) is a generalization of the naïve sampler model that adds noise and sensitivity to the average payoffs. The current predictions of SAW were derived with the parameters estimated in Erev, Ert, Plonsky, and Roth (2020). Table 1 shows that the Cognitive Hierarchy model predicts that the Alert app will be as effective as the Enforcing app, but the reliance on small samples

${ }^{2}$ While it is hard to know which past experiences agents consider "similar", the small sample of similar past experiences can be approximated well by assuming that agents rely on small random samples of past experiences. This assumption has been shown to predict behavior well in recent choice prediction competitions (Erev et al., 2017; Plonsky et al., 2019) 
models predict that only the Enforcing app will be effective. The experiment described below was designed to test these predictions.

\section{Experimental study}

\section{Method}

One hundred fifty-eight MTurk workers participated in the experiment in exchange for monetary compensation. Each session included one of the three conditions: Basic $(n=48,12$ groups, 30 males; $\left.M_{\text {age }}=41\right)$, Alert $\left(n=58,15\right.$ groups, 36 males $\left.^{3} ; M_{\text {age }}=34\right)$ or Enforcement $(n$ $=52,13$ groups, 31 males, $M_{\text {age }}=36$ ). Each participant could participate in only one of the sessions. The monetary payoff included a show-up fee of \$1 and an additional guaranteed \$2 if the participant made more than $66 \%$ of the choices (i.e., more than 40 out of the 60 choices) on time $^{4}$ as well as a chance to earn $\$ 1$ bonus, based on the number of points accumulated during the experiment (mean final pay $=\$ 3.35)$.

The experiment was run in groups of four participants. Participants could proceed to the next round only after all four players made their choices. To ensure that the experiment runs smoothly, we told participants that they had 12 seconds (20 seconds in the first three trials) to make their choice in each round, after which the program will automatically submit a choice for them, and they will receive a penalty of 2 points. Unbeknownst to participants, when the program auto submitted a choice on their behalf, it made the same choice the participant did in the previous trial (in the first trial the program auto-submitted the Responsible choice) ${ }^{5}$

The experiment, programmed with OTree (Chen et al., 2016), employed a variant of the clicking paradigm (Barron \& Erev, 2003). In each of 60 trials, the participants deliberated between two keys " $A$ " and "B". They saw a complete description of the incentives structure and after each trial received feedback regarding their obtained and forgone payoff (see Figure 1).

${ }^{3}$ Four participants had missing age and gender.

${ }^{4}$ The instructions said that the $\$ 2$ will be received if the subject made "most of the choices" on their own.

${ }^{5}$ Originally there were 272 participants. The analysis focuses on the choices made by the participants when they responded by the time limit (12 seconds in most trials). Groups in which more than $20 \%$ of the choices were not made by the time limit (and the computer repeated the last choice) were not included in the analysis at all. The reason for the very high exclusion rate is coordination: often the participants have to wait for a certain amount of time before other participants enter the groups. It is common that by the time the group is formed, the first participant already voluntarily dropped out the experiment. Including all groups (or using other cutoff rather than the $20 \%$ ) does not meaningfully change the mean Reckless-rates. 
1 Figure 1: Screens presented to participants, in 'Reckless or Responsible game, "Alert" app condition

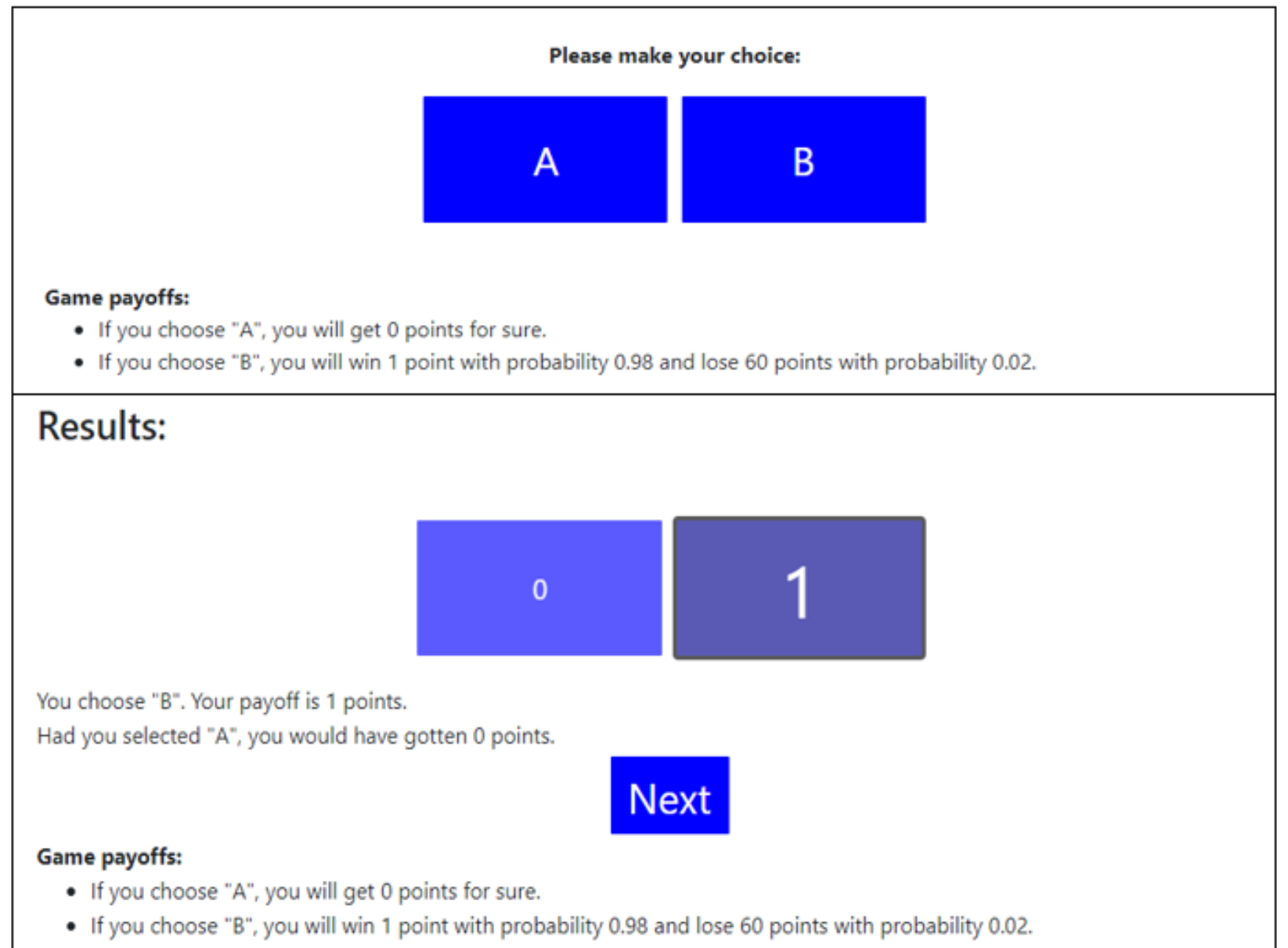

Note. The upper image ("Please make your choice") presents the screen at the beginning of each trial. The lower image ("Results") presents the screen at the end of each trial.

Results

The right-hand column in Table 1 presents the mean choice rate of the reckless option (Recklessrate) in the three conditions. The rates are $91 \%, 92 \%$ and $15 \%$ in the Basic, Alert, and

11 Enforcement conditions, respectively. That is, the Alert app was ineffective, while the Enforcement app was highly effective in reducing the Reckless-rate. The difference between 13 Basic and Alert is insignificant, $t(104)=0.53$. The difference between Enforcement and Basic 14 condition is significant, $t(98)=16.98, p<.001$, and so is the difference between Enforcement 15 and Alert, $t(108)=18.48, p<.001$.

Figure 2 presents the effect of experience on each participant within the first 10 groups,

18 equilibrium. Analysis of the choice rate in the very first trial reveals a pattern that was not

19 predicted by the current models. The initial reckless-rates are $73 \%, 81 \%$ and $19 \%$ in the Basic,

20 Alert, and Enforcement conditions, respectively. Under one post-hoc explanation of this pattern 
1 it reflects overgeneralization from (a small sample of) similar real-life situations that occurred before the beginning of the current experiment (Plonsky et al., 2015).

Figure 2: Individual and Group level 'Reckless choice rates in the "Basic" (up), "Alert" (middle) and Enforcing (down) conditions. Only first 10 groups in each condition, and the mean over all groups in that condition, are shown
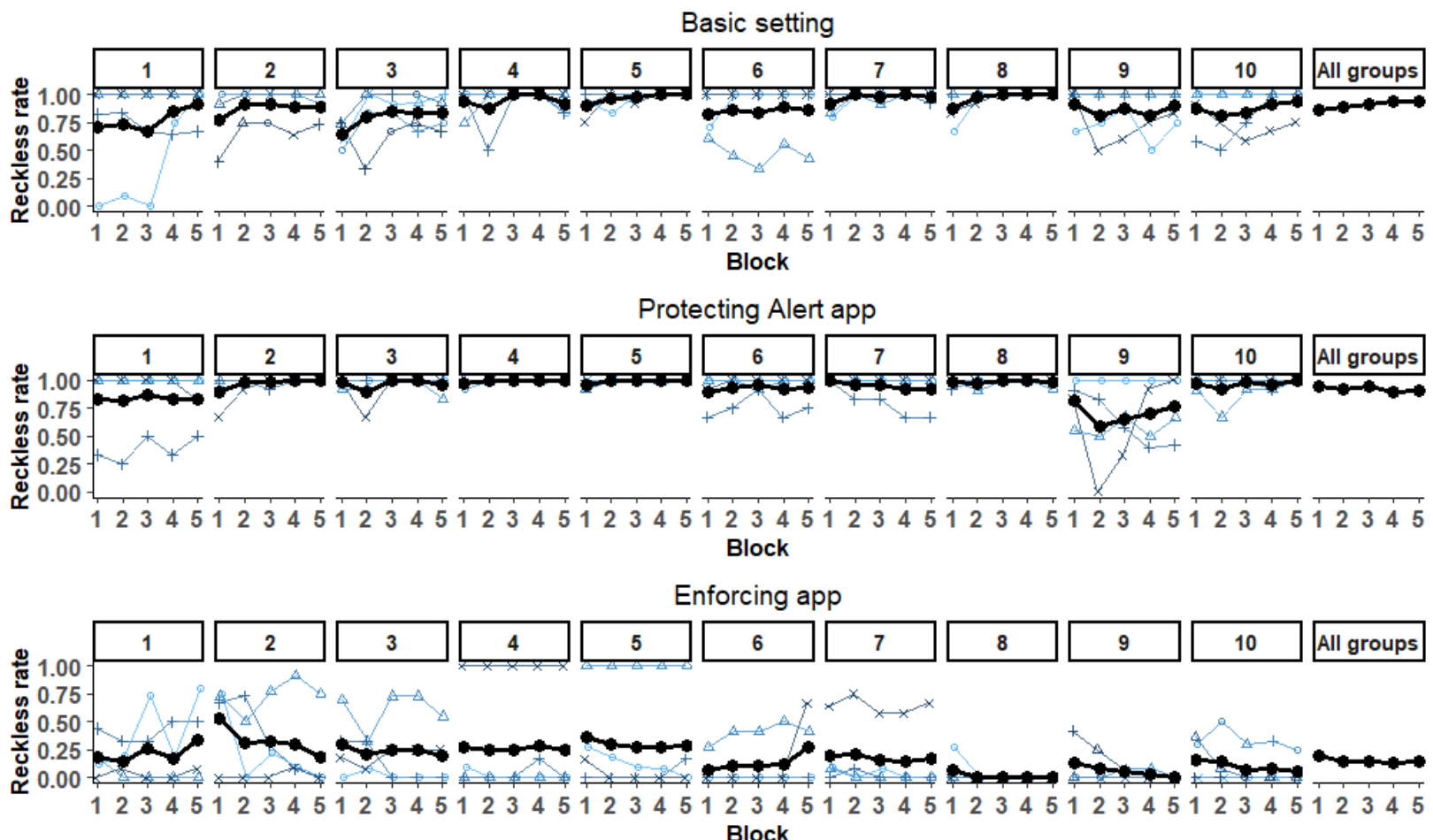

Note. Each line represents a Reckless choice rate in five blocks (of 12 trials) by a subject in the respective group, in a respective condition. The bold line shows the mean Reckless rate of the respective group. The rightmost plot (of each condition) present the overall mean Reckless rate of the respective condition.

\section{General Discussion}

The COVID-19 pandemic presents major challenges to individuals and to policy makers. Individuals try to strike a balance between reducing their personal risk of infection and minimizing the social and economic costs of safe behavior. Policy makers face similar challenges on a larger scale - trying to balance public health with economic and civil considerations. Harsh interventions, like the aggressive use of tracking applications in China appear to be effective. Yet, such interventions have downsides, as they compromise citizens' freedom of choice and impose a threat to personal privacy.

The current paper explored two approaches to the design of policies that imply less aggressive use of tracking applications. The first-Alert only apps-avoids enforcement altogether and focuses on protecting the users from the reckless behavior of other agents via alert systems. In the current setting, this approach should be effective if users are rational or deviate from rationality in three well studied manners: risk aversion, oversensitivity to rare losses (as predicted by cumulative prospect theory, Tversky \& Kahneman, 1992), or cognitive hierarchy 
(Camerer et al., 2004). The second approach-Gentle Enforcement apps-is predicted to be effective even if the agents base their decisions on small samples of similar past experiences. Our experimental results show that in the current setting, focusing exclusively on alerts is not effective at all, but gently enforcing the rules is highly effective.

We abstract our experiments as a simplification of repeated decisions in a time of a pandemic, but participants were unaware of this abstraction when they made their decisions. We can therefore generalize these results to other similar settings. Specifically, the results show that in the presence of rare detrimental events, minimizing negative social externalities that reluctantly lead sophisticated agents to take more risk is insufficient. Even when social externalities are eliminated altogether, most individuals behave as if they underweight rare events; to discourage such detrimental risk-taking, it is necessary to reduce the common benefit from taking that risk (Erev, Gilboa-Freedman \& Roth, 2019).

In order to clarify the negative implications of the current analysis it is important to recall that in the first half of 2020, policy makers in Western democracies tried to facilitate the development of tracking applications that focused on alerts, and tried to avoid enforcing applications. Part of the justifications of this bias involves the assertion that good alert systems are expected to be effective under rational economic theory, and also under the best known deviations from rational choice. The present analysis questions the value of this justification. It suggests that in the current setting, the reliance on small samples hypothesis captures the main deviations from rational choice. Another justification for focusing on Alert-type apps is that such apps appear intuitively more likely to be adopted by the population as they can be designed using high privacy-protection principles. However, computational analysis of agents who rely on small samples shows that using a clever design, gentle rule enforcement apps can have even higher adoption rates in the population (Plonsky \& Erev, 2020)

The positive implications of the present investigation involve the potential of gentle rule enforcement. To illustrate this potential, we chose to conclude with an example demonstrating the difference between "aggressive privacy invading enforcement" and "gentle rule enforcement" apps. Consider the rule "people that leave neighborhood X should be quarantined for 14 days," and assume that a tracking app detects entry to this neighborhood. An aggressive app can record the data, and punish the user with 14-days quarantine when the user leaves X. A gentle rule enforcement app achieves similar impact by sending a message of the following type: "You have just entered neighborhood X, and if you will not leave in less than 10 minutes, this fact will be recorded, and you will lose the Green code." $" 6$ Thus, if the user leaves in time, nothing is recorded, and the sole punishment is the requirement to leave the problematic neighborhood. We believe that policy makers should facilitate the development of gentle rule enforcement apps of this type. While the present analysis does not prove that gentle rule enforcement apps will be successful, it suggests that they can be highly effective.

\footnotetext{
${ }^{6}$ This 10-minute grace rule is meant to minimize false alarms from coming near restricted zones. Such false alarms are expected to hurt the common benefit from using the app and impairs the idea of gentle rule enforcement. 


\section{References}

Barron, G., \& Erev, I. (2003). Small feedback-based decisions and their limited correspondence to description-based decisions. Journal of Behavioral Decision Making, 16(3), 215-233.

Camerer, C. F., Ho, T. H., \& Chong, J. K. (2004). A cognitive hierarchy model of games. The Quarterly Journal of Economics, 119(3), 861-898.

Chen, D. L., Schonger, M., \& Wickens, C. (2016). oTree-An open-source platform for laboratory, online, and field experiments. Journal of Behavioral and Experimental Finance, 9, 88-97.

Costa-Gomes, M., Crawford, V. P., \& Broseta, B. (2001). Cognition and behavior in normalform games: An experimental study. Econometrica, 69(5), 1193-1235.

Erev, I., Ert E., Plonsky O., \& Roth, Y (2020). Six Contradicting Deviations from Rational Choice, and the Impact of Experience, working paper.

Erev, I., Ert, E., Plonsky, O., Cohen, D., \& Cohen, O. (2017). From anomalies to forecasts: Toward a descriptive model of decisions under risk, under ambiguity, and from experience. Psychological review, 124(4), 369-409.

Erev, I., Gilboa Freedman, G., \& Roth, Y. (2019). The impact of rewarding medium effort and the role of sample size. Journal of Behavioral Decision Making, 32(5), 507-520.

Erev, I., Ingram, P., Raz, O., \& Shany, D. (2010). Continuous punishment and the potential of gentle rule enforcement. Behavioural Processes, 84(1), 366-371.

Erev, I., Plonsky O., \& Roth, Y (2020). The impact of rare disasters, and the value of gentle rule enforcement in addressing pandemics, working paper.

Erev, I., \& Roth, A. E. (2014). Maximization, learning, and economic behavior. Proceedings of the National Academy of Sciences, 111(Supplement 3), 10818-10825.

Huang, Y., Sun, M., and Sui, Y. (2020). How Digital Contact Tracing Slowed Covid-19 in East Asia. Harvard Business Review, April 15, Retrieved from https://hbr.org/2020/04/how-digitalcontact-tracing-slowed-covid-19-in-east-asia.

Kahneman, D., \& Tversky, A. (1979). Prospect Theory: An Analysis of Decision under Risk. Econometrica, 47(2), 263-292.

Nagel, R. (1995). Unraveling in guessing games: An experimental study. The American Economic Review, 85(5), 1313-1326. 
1 Plonsky, Ori, Reut Apel, Eyal Ert, Moshe Tennenholtz, David Bourgin, Joshua C. Peterson, 2 Daniel Reichman et al. "Predicting human decisions with behavioral theories and machine 3 learning." arXiv preprint arXiv:1904.06866 (2019).

Plonsky O. \& Erev, I., (2020). To get people to adopt tracing applications, minimize the probability they will regret it. working paper.

Plonsky, O., Teodorescu, K., \& Erev, I. (2015). Reliance on small samples, the wavy recency 9 effect, and similarity-based learning. Psychological review, 122(4), 621-647.

Roth, Y., Wänke, M., \& Erev, I. (2016). Click or Skip: The role of experience in easy-click checking decisions. Journal of Consumer Research, 43(4), 583-597.

Schurr, A., Rodensky, D., \& Erev, I. (2014). The effect of unpleasant experiences on evaluation and behavior. Journal of Economic Behavior \& Organization, 106, 1-9.

Stahl, D. O., \& Wilson, P. W. (1995). On players' models of other players: Theory and experimental evidence. Games and Economic Behavior, 10(1), 218-254. of uncertainty. Journal of Risk and Uncertainty, 5(4), 297-323. 


\section{Appendix}

3 The Cognitive Hierarchy model (CH, Camerer, Ho \& Chong, 2004)

$4 \mathrm{CH}$ assumes that each agent is of one of k types. Agent of type Step-0 (or level-0) randomize;

5 and Step- $k$ thinkers best-respond, assuming that other players are distributed over Step-0 through

6 Step- $k-1$ categories. The shape of the assumed distribution of type is Poisson. Camerer et al.

7 estimated the single parameter of the Poisson distribution in large number of games and suggest

8 that "assuming $\tau$ value of 1.5 could give reliable predictions for many other games as well."

9 This value implies that $22 \%$ of the agents are Step- 0 .

\section{The naïve sampler model (Barron \& Erev, 2003)}

12 The model assumes random choice in the first trial $(t=1)$, and sampling-based decisions in

13 subsequent trials. The decision at $\mathrm{t}>1$ starts by randomly sampling $\kappa$ previous trials with 14 replacement ( $\kappa$, a positive integer, is a free parameter). Then, the option with the largest average 15 payoff in the sample is selected. Following Erev and Roth (2014) the current implementation 16 assumes $\kappa=5$.

\section{The Sampling and Weighting (SAW) model (Erev et al., 2020)}

SAW generalizes the Noisy naïve model. It assumes two response modes: exploration that implies random choice and exploitation that implies reliance on samples of 5 past experience (like the naïve sampler model). The probability of exploration at trial $t$ is: $\varepsilon^{(t-1) / t}$.

In addition, SAW assumes that the estimation of the value of Option $\mathrm{j}$ under the exploitation mode is a weighted average of two estimates: Grand Mean $\left(\mathrm{GM}_{\mathrm{j}, \mathrm{t}}\right)$ - the option's mean payoff in the previous $\mathrm{t}-1$ trials, and sample mean $\left(\mathrm{SM}_{\mathrm{j}, \mathrm{t}}\right)$ - the option's mean payoff in a sample of $k_{i}$ past trials:

$$
\text { Evaluation }_{\mathrm{j}, \mathrm{t}}=\mathrm{w}_{\mathrm{i}}\left(\mathrm{GM}_{\mathrm{j}, \mathrm{t}}\right)+\left(1-\mathrm{w}_{\mathrm{t}}\right) \mathrm{SM}_{\mathrm{j}, \mathrm{t}}
$$

The weight of the grand mean $\mathrm{w}_{\mathrm{i}}$ is 0.5 with probability $\omega$ (a free parameter), and 0 otherwise. The value of $\mathrm{k}_{\mathrm{i}}$ is uniformly drawn from the set $\{1,2 \ldots \kappa\}$ where $\kappa$ is a free parameter. The current predictions were derived with the parameters $\kappa=9, \varepsilon=0.4$, and $\omega=0.5$. These values best fit the 88 conditions analyzed in Erev, Ert, Plonsky, and Roth (2020). 